\title{
New method for the discrimination of single-source events in heavy-ion collisions
}

P. Désesquelles ${ }^{1, b}$, A.M. Maskay ${ }^{5}$, P. Lautesse $^{5}$, A. Demeyer ${ }^{5}$, E. Gerlic ${ }^{5}$, D. Guinet $^{5}$, M. Stern ${ }^{5}$, J.L. Laville ${ }^{1}$, G. Auger ${ }^{1}$, Ch.O. Bacri ${ }^{2}$, L. Beaulieu ${ }^{9}$, N. Bellaize ${ }^{3}$ F. Bocage ${ }^{3}$ B. Borderie ${ }^{2}$, R. Bougault ${ }^{3}$, B. Bouriquet ${ }^{1}$, R. Brou ${ }^{3}$, P. Buchet ${ }^{4}$, J. Colin $^{3}$, N. De Cesare $^{7}$, D. Doré ${ }^{4}$, D. Durand ${ }^{3}$, J.D. Frankland ${ }^{1}$, P. Gagné ${ }^{9}$, E. Galichet ${ }^{2,8}$, E. Genouin-Duhamel ${ }^{3}$, M. Germain ${ }^{c}$, S. Hudan ${ }^{1}$, R. Laforest ${ }^{9}$, F. Lavaud ${ }^{2}$, J.F. Lecolley ${ }^{3}$, C. Leduc ${ }^{5}$, R. Legrain ${ }^{4}$, N. Le Neindre ${ }^{3}$, O. Lopez ${ }^{3}$, M. Louvel ${ }^{3}$, L. Nalpas $^{4}$, J. Normand ${ }^{3}$, M. Pârlog ${ }^{6}$, M.F. Rivet ${ }^{2}$, E. Rosato ${ }^{7}$, R. Roy ${ }^{9}$, F. Saint-Laurent ${ }^{1, a}$, J.C. Steckmeyer ${ }^{3}$, G. Tăbăcaru ${ }^{6}$, B. Tamain ${ }^{3}$, L. Tassan-Got ${ }^{2}$, O. Tirel ${ }^{1}$, E. Vient ${ }^{3}$, M. Vigilante ${ }^{7}$, C. Volant ${ }^{4}$ J.P. Wieleczko ${ }^{1}$, (INDRA collaboration)

${ }^{1}$ GANIL, CEA et IN2P3-CNRS, B.P. 5027, F-14076 Caen Cedex, France.

${ }^{2}$ Institut de Physique Nucléaire, IN2P3-CNRS, F-91406 Orsay Cedex, France.

${ }^{3}$ LPC, IN2P3-CNRS, ISMRA et Université, F-14050 Caen Cedex, France.

${ }^{4}$ DAPNIA/SPhN, CEA/Saclay, F-91191 Gif sur Yvette Cedex, France.

${ }^{5}$ Institut de Physique Nucléaire, IN2P3-CNRS et Université, F-69622 Villeurbanne Cedex, France.

${ }^{6}$ National Institute for Physics and Nuclear Engineering, RO-76900 Bucharest-Măgurele, Romania.

7 Dipartimento di Scienze Fisiche e Sezione INFN, Università di Napoli "Federico II", I80126 Napoli, Italy.

${ }^{8}$ Conservatoire National des Arts et Métiers, F-75141 Paris cedex 03.

${ }^{9}$ Département de physique. Université Laval, Qubec, Canada G1K 7P4.

a) present address: DRFC/STEP, CEA/Cadarache, F-13018 Saint-Paul-lez-Durance Cedex, France.

b) permanent address: Institut des Sciences Nucléaires de Grenoble, IN2P3-CNRS et Uni- 
versité Joseph Fourier, F-38026 Grenoble Cedex, France c) present address: IRes, 23 rue du Loess, 67037 Strasbourg CEDEX 2, France.

\begin{abstract}
This paper introduces a new method for the selection of central singlesource events, based on classical multivariate techniques. The resulting discriminating variable is shown to be valid for different hypotheses on the nuclear source deexcitation mechanism. It enables the selection of events which are representative of the whole set of single-source events. Application to the $\mathrm{Ni}+\mathrm{Ni}$ at $32 \mathrm{~A} \mathrm{MeV}$ system measured with the INDRA multidetector has allowed the determination of the fusion probability as a function of the impact parameter and the evaluation of the corresponding cross-section.
\end{abstract}

\title{
I. INTRODUCTION
}

In spite of a large number of analyses, it is still a matter of discussion up to what heavy ion beam energy, thermalized single-source events persist. By single-source events, we mean collisions in which, after non-equilibrium phases [1,2], a thermalized fused system is formed. Interest in fusion is due to the fact that this mechanism corresponds to one of the most fundamental rearrangements of nuclear matter and, furthermore, leads to the formation of maximally hot and dense single nuclear sources. Over the so-called intermediate beam energy range, most of the observed cross-section corresponds, at least for heavy systems [3], to dumbbell shape sources. However, it has been recently demonstrated that, for central collisions, most of the mass of a heavy system can be concentrated in a hot compact shape single-source (Xe+Sn at $50 A \mathrm{MeV}[4], \mathrm{Gd}+\mathrm{U}$ at $36 A \mathrm{MeV}[5], \mathrm{Au}+\mathrm{Au}$ at $35 A \mathrm{MeV}[6])$. This single source deexcites, after a pre-equilibrium phase, in times much shorter than those 
involved for the classical fusion-fission process. Thus, in the following we will refer to it as fusion-like events. In this paper, we report on a recent work performed on the system ${ }^{58} \mathrm{Ni}+{ }^{58} \mathrm{Ni}$ at $32 \mathrm{~A} \mathrm{MeV} \mathrm{[7],} \mathrm{i.e.,} \mathrm{in} \mathrm{a} \mathrm{centre} \mathrm{of} \mathrm{mass} \mathrm{energy} \mathrm{domain} \mathrm{where} \mathrm{the} \mathrm{fusion} \mathrm{cross-}$ section is expected to be small compared to the reaction cross-section [8]. In this beam energy range, higher impact parameters lead to binary (quasi-target + quasi-projectile) events due both to the smaller mean free path and to the decrease of the density with the distance to the nucleus centre. The mean impact parameter leading to fusion decreases with the increase of the bombarding energy because of the raise of the centrifugal force and of the weakening of the stopping power (n-n cross-section or nuclear viscosity) [8]. Furthermore, the fusion-like scenario is complicated by the increasing number of pre-equilibrium particles.

This paper is concerned with the selection of events corresponding to a single nuclear source. We discuss here a new protocol based on the so-called Discriminant Analysis statistical method [9-11], applied to the mass/kinetic energy quadri-moments (Sec. II A 3). This technique will be shown to provide a variable which has a high discriminating power (Sec. II A 2) and representativeness (Sec. II C). The method used to isolate single-source events has been tested using the Simon [12,13], SMm [14] and Gemini [15] codes as event generators. It will be shown that the cut off technique applied to global variables commonly used for heavy system single-source selection is not efficient for small systems (Sec. II B 1). The protocol enables the measurement of the fusion-like cross-section and a reliable selection of single-source events. Finally, this selection technique will be applied to INDRA [16] experimental data [7] (Sec. III). 


\section{DISCRIMINATION TECHNIQUE}

\section{A. Mathematical tools}

\section{Error rate and Overlap $\mathcal{O}$}

The most natural way to define the two-category discrimination associated with a variable is to choose a threshold value which separates the events attributed to the first category from the events attributed to the second one. The error rate is defined as the proportion of badly attributed events. Its value goes from zero, when the two category distributions are completely separated, to 0.5 , when the distributions are super-imposed, and to 1 , when the distributions are separated but the events systematically misattributed. Hence the error rate depends also on the position of the threshold : the minimum error rate is obtained when the threshold is fixed at the crossover of the two distributions, whatever their shapes. In this case, the error rate is simply the overlap integral divided by the total number of events. In the following, this minimum error rate will be referred to as the overlap $\mathcal{O}$.

\section{Discrimination Power $\lambda$}

The criterion traditionally used in statistics to measure the separation between two categories is the discrimination power. This quantity is large when the distance between the categories is large and their widths are small. The latter term is given by the intra-category variance $V^{\text {intra }}=n_{1} \sigma_{1}^{2}+n_{2} \sigma_{2}^{2}$ where the $n_{i}$ are the number of events in each category and the $\sigma_{i}$ are their standard deviations. Let $G, G_{1}$ and $G_{2}$ be, respectively, the total, first and second category mean values. The inter-category variance, measuring the distance between

the categories, is defined as $V^{\text {inter }}=n_{1}{\overline{G G_{1}}}^{2}+n_{2}{\overline{G G_{2}}}^{2}$. Since $V^{\text {inter }}+V^{\text {intra }}=V^{\text {tot }}$ (the total variance), the discrimination power can be formulated as :

$$
\lambda=\frac{V^{\text {inter }}}{V^{\text {tot }}}
$$


This quantity belongs to the interval $[0,1]$. It is equal to zero when the two distributions have the same mean value $\left(G=G_{1}=G_{2}\right)$ and to one when the mean values are different and all the events of a given category have the same value (so that $\sigma_{1}=\sigma_{2}=0$ ).

In order to generate such optimum cut-off variables, a statistical technique referred to as Discriminant Analysis (D.A.) is commonly used in many domains [9,10,17]. Starting from a set of observables $o_{j}$, the D.A. computes the set of coefficients $\alpha_{j}$ such that, the discriminating variable $d=\sum_{j} \alpha_{j} o_{j}$ has the maximum discrimination power.

\section{Multivariate moments}

The next step consists in choosing the set of observables to which the D.A. will be applied. This choice will be made, in the two next sections, following two logics : either one uses an ensemble of variables which are a priori known to provide a good discrimination (examples are given in the following). Or one can try to define a set of variables which summarizes the whole experimental information.

In most heavy ion collision experiments, the information related to each detected reaction product can be formalized by a four dimensional vector formed by the mass $A$ of the ion and the coordinates $v_{x}, v_{y}, v_{z}$ of its velocity vector. In order to avoid unit problems, the four components may be replaced by their energetic equivalents, that is by $\left(E_{A}, E_{x}, E_{y}, E_{z}\right)$ where $E_{A}$ is the mass energy and $E_{i}=E_{k i n} \cos \theta_{i}\left(E_{k i n}\right.$ being the kinetic energy in the laboratory frame and $\theta_{i}$ the angle between the detector which was hit and axis $i, i \in\{x, y, z\}, z$ being the beam axis). Since we are interested in the discrimination of events it is necessary to define global variables holding faithfully the whole event information. A convenient choice is the quadri-moments defined as :

$$
M_{j k l m}=\sum_{\nu=1}^{M} E_{A}^{j}(\nu) E_{x}^{k}(\nu) E_{y}^{l}(\nu) E_{z}^{m}(\nu)
$$

where $\nu$ is the fragment (or light particle) number of the event with multiplicity $M$. It can be shown [7] that the moments known up to high enough $(j, k, l, m)$ orders allow to 
reconstruct the initial fragment characteristics. In our case, the orders run from 0 to 4 (625 variables, if the orders run up to 5, the results suffer from numerical bias). Indeed, low order moments are less affected by statistical fluctuations and identification uncertainties and contain most of the information. In order to illustrate this latter point, the proportion of the total $6^{4}$ moment information given by the first $n^{4}(n \leq 6)$ moments, as calculated by the Principal Component Analysis technique $[9,18]$, is plotted in figure 1 . As can be seen, the saturation of the statistical information (see references $[9,11]$ ) with the number of moments is already reached, i.e. $625\left(5^{4}\right)$ moments provide almost the same amount of statistical information as $1296\left(6^{4}\right)$ moments.

\section{B. Application of the multivariate analysis techniques}

\section{Global variables commonly used for discrimination}

Let us examine now the first possible choice for the initial set of observables. The discrimination efficiency of the variables being highly dependent on the system and the incident energy under study, the overlap and the discrimination power of a large set of these variables have been calculated in the $\mathrm{Ni}+\mathrm{Ni}$ at $32 \mathrm{~A} \mathrm{MeV}$ case. For this purpose, we need events for which the single- or poly-source nature is known. Such events can only be obtained using simulations. We have checked that a sample of events produced by the Simon event generator code $[12,13]$ over the whole impact parameter range was in good agreement with the experimental sample, after taking into account the experimental apparatus filtering effect and using the same selection criteria (the total detected charge is at least $80 \%$ of the system charge and the total detected linear momentum is at least $80 \%$ of the system linear momentum). These completeness criteria select by themselves events corresponding to low impact parameters $(b<6 \mathrm{fm}$, for Simon events). Any extra selection on the multiplicity would also reject single-source events, that is modify the representativeness of the final singlesource sub-sample. Some illustrations of the concordances between experimental and Simon 
samples are displayed in figure 2. Most static, kinematic and shape variable distributions are reasonably well reproduced, so that the Simon code can be used as event generator in the following.

Some variables, which are known to provide good discrimination for heavy systems $[6,19,20]$ appear to have much less efficiency for lighter ones. Most of these variables characterize the shape of the events which, for single-sources, is expected to tend towards a sphere in velocity space. For light single source systems, due to the reduced multiplicity, these variables cannot reach the values corresponding to spherical shapes [20,21]. The values of the discrimination power and the overlap for our selected sample of complete events from the $\mathrm{Ni}+\mathrm{Ni}$ system at $32 \mathrm{~A} \mathrm{MeV}$ are given in Table I for a set of 22 variables as defined in the table's references. The variable that gives the best - though still insufficient - discrimination is the isotropy ratio, defined here as :

$$
I=\frac{\sum_{\nu} p_{\perp}(\nu)}{\sum_{\nu} p(\nu)}
$$

where $p(\nu)$ is the modulus of the center of mass linear momentum of the reaction product number $\nu$ and $p_{\perp}(\nu)$ its component perpendicular to the beam direction. More generally, the variables that give the best discriminations in Table I $\left(I, \Theta_{\text {flow }}, E_{\text {rat }}\right)$ are connected with the proportion of fragment emission perpendicular to the beam. The fact remains that none of these variables allows a clear selection of a single-source set of events [7].

A D.A. performed on the set of 22 variables provides only a relatively small improvement with respect to the isotropy ratio values, since the overlap is reduced by a factor of $12 \%$ and the discrimination power increased by a factor of $7 \%$ (see $d_{g l o b}$ in Table I). As mentioned before, this is mainly due to the fact that many of the variables chosen here mostly characterize the shape of the source in velocity space. Together with the conservation rules, this explains why they give very correlated information. 


\section{Multivariate moments}

The second way to proceed, i.e. application of the D.A. to the quadri-moments, leads to quite different results (see figure 3). Most individual moments provide a very small amount of discrimination $(\lambda \approx .001)$. The relative best results $(\mathcal{O}<0.35, \lambda>0.1)$ are obtained by the moments of the type $M_{j 00 m}=\sum_{\nu} E_{A}^{j}(\nu) E_{z}^{m}(\nu)$ that is the moments measuring (weighted) elongations along the beam axis (but note that $\mathcal{O}>0.17$ and $\lambda<0.32$ for each of them).

In spite of the poor discrimination brought by the moments taken individually, even the best ones, the corresponding discriminating variable, given by discriminant analysis (II A 2) and which reads :

$$
d_{625}=\sum_{(j, k, l, m)=0}^{4} \alpha_{j k l m} M_{j k l m},
$$

appears definitely much more efficient than the one found in the previous analysis $\left(d_{g l o b}\right)$, since $\lambda\left(d_{625}\right)=0.78$ and $\mathcal{O}\left(d_{625}\right)=0.035$ (see Fig. 3 ). In this case, many moments carry independant statistical information and account for the fine correlations induced by the conservation laws or for the fragment interactions which may be very different for singleand poly-source events.

In the following we will be interested in checking that the experimental single-source events selected using $d_{625}$ actually correspond to low impact parameter collisions. As $b$ cannot be directly measured, we need to define an estimate.

\section{Estimate of $b$ : chimera variable}

Another multivariate analysis technique allows the calculation of the linear combination of a set of observables which gives the best estimate of a hidden (not directly measurable) parameter. This linear combination is sometimes called the chimera of the hidden parameter [9]. We shall use this quantity to estimate the impact parameter $b$. With the help of a Simon simulation, the chimera ${ }^{c} b$ of the impact parameter was calculated from the first 81 $\left(3^{4}\right)$ quadri-moments : 


$$
{ }^{c} b=\sum_{(j, k, l, m)=0}^{2} \beta_{j k l m} M_{j k l m}
$$

so that the residue $\sum_{\text {Simon events }}\left(b-{ }^{c} b\right)^{2}$ is minimum.

The correlation between the impact parameter and its chimera is attested in figure 4 (linear correlation coefficient $\rho=0.90$ ) for $\mathrm{Ni}+\mathrm{Ni} 32 \mathrm{~A} \mathrm{MeV} \mathrm{Simon} \mathrm{events.} \mathrm{Then,} \mathrm{figure} \mathrm{5,}$ which displays the correlation ${ }^{c} b=f\left(d_{625}\right)$ lets appear the clear discrimination feature of the two bumps corresponding to the single-source (lower left) and poly-source (upper right) events.

\section{Representativeness}

Whatever the variable used to isolate single-source events, only a given proportion of these events will be clearly separated from the poly-source ones. Discrimination power and overlap allow to quantify the fraction of well separated events. It is then necessary to qualify the representativeness of the selected sample of events, i.e., evaluate to what extent the characteristics of the selected events are similar to those of the whole set of singlesource events. Once again, this means resorting to simulated events. We have compared the well separated events, i.e., the events situated under a threshold allowing a $5 \%$ pollution from poly-source events, to the complete set of Simon single-source events. The results are presented in Fig. 6 where the distributions for the selected single-source events are normalized to the total number of generated single-source events. When the discriminating variable $d_{625}$ is used $\left(d_{625}<-0.04\right)$, no observable distribution is altered by the selection (see figure 6). This is not true for all observables when the same selection ( $5 \%$ pollution) is made using the isotropy ratio, particularly as regards the orientation of the events (Fig. 6d)).

\section{Verification with other models}

Besides the reliability of the selection, another aspect concerning the relevance of the discriminating variable $d_{625}$ has to be checked [7]. Actually, $d_{625}$ has been obtained with Simon. 
Thus, it has to be verified that this variable can also recognize single and poly source samples generated by other deexcitation models and pre-equilibrium treatments. Thus samples have been generated using the DBS ( [33] and references herein) event generator which falls into three steps. First, an entrance channel code simulates the pre-equilibrium emission (the numbers of protons $n_{p}$, neutrons $n_{n}$, and their mean kinetic energy $T$ are treated as parameters). Next, DBS determines whether the event is single or bi-source depending on the impact parameter (classical trajectory model) and calculates the mass, charge and excitation energy of the sources. The last step consists of the simulation of the deexcitation of the sources using either the Gemini [15] (simulation of the disintegration of a hot nucleus via a sequence of binary splittings) or the SMM [14] (instantaneous statistical multifragmentation of a hot nucleus) codes. The distributions of the $n_{p}, n_{n}$ and $T$ parameters are determined using the backtracing technique [34] in order to obtain an optimum reproduction of the experimental charge partitions. In all cases the $d_{625}$ distributions (using the $\alpha$ given by Simon) lie in the same range as for Simon as shown in figure 7. In the case of the single source events, the shift of the maximum and the spread of the right hand tail for DBS + Gemini and DBS + SMM are due to the pre-equilibrium parameter distributions. Hence the $d_{625}$ variable appears to be only weakly sensitive to the disintegration mechanism in that sense that single(poly) source distributions are always on the left(right) side of the $d_{625}$ range.

The discriminating variable may now be confidently applied to experimental data.

\section{DETERMINATION OF THE FUSION CROSS-SECTION}

The experimental events were analysed in the 625 dimensional space. However, as experimental clouds might not occupy exactly the same positions as the simulated clouds, an even better separation axis can be searched for. This was done by imposing small deviations to the $d_{625}$ axis evaluated with Simon and by testing systematically the resulting discrimination. The optimisation was realized using the POWELL minimisation routine from the Numerical Recipes [35]. The criterium for optimum discrimination corresponds to a 
maximum separation between the two components of the $d_{625}$ distribution (see figure $\left.9 \mathrm{a}\right)$ ). Indeed, the routine converged rapidely towards an axis which is very close to the Simon one (with a correlation coefficient between the two discrimination axes $\rho=0.997$ ) but which still improved the separation, showing, once more, the adequacy of the Simon code. Different starting points were tested in the vicinity of the Simon axis. They all lead to the same resulting experimental axis. This shows the relative independance of the final results to the chosen model generator.

Finally, the experimental events (after completeness selection) are presented in figure 8. The horizontal axis is the optimum discrimination variable and the vertical axis the chimera impact parameter calculated using formula (5) with the $\beta$ parameters given by the Simon code. The range of the chimera variable is the same for the experimental events as for the Simon events which is an indication that it is also a good experimental estimate of the impact parameter.

Two bumps can be observed at locations which are close to the Simon ones (Fig. 5). The lower left one is attributed to the single-source events. The right shift of the single-source bump is due to the fact that experimental events appear to be less spherical on average than Simon events [7]. Furthermore, the single-source bump appears to be more prominent in the experimental case which is a first indication that the model fusion cross section may be underestimated. Although the detailed validation of the Simon code is not the purpose of the present paper, the agreement between figures 5 and 8 strengthens the reliability of this event generator. The experimental distribution of the $d_{625}$ variable (Fig. 9 top) shows a two component distribution (which was the case neither for the global variables nor for $\left.d_{g l o b}\right)$. The $d_{625}$ experimental distribution appears to be nicely represented by the sum of two Gaussians (Fig. 9 top). This result is not surprising considering that each bump corresponds to the convolution of 625 distributions (even though, of course, all these distributions are not independant). Besides the two distributions corresponding to the Simon single- and poly- source events are also well reproduced by Gaussians. In the following, we will consider the two Gaussians as the distributions for single- and poly-source events. 
In order to estimate the fusion-like cross section, we will deduce the experimental impact parameter distribution for single-source events from the experimental $d_{625}$ distribution (the chimera impact parameter is no longer used in the following). When the differential event efficiency $\epsilon(b)$ of the detector filter and of the selection conditions is known (in our case, it is given by Simon), the impact parameter determination technique used for example in Ref. [36] can be improved in order to obtain $b$ on an absolute scale. The impact parameter probability distribution of the experimental events is :

$$
P(b)=\frac{\epsilon(b) b}{\int_{o}^{\infty} \epsilon\left(b^{\prime}\right) b^{\prime} \mathrm{d} b^{\prime}}
$$

The assumption that the events are ordered in the same way along the $d_{625}$ and the $b$ axes can be written in mathematical terms using the distribution functions: $F(b)=F\left(d_{625}\right)$, i.e.,

$$
\int_{0}^{b} \frac{\epsilon\left(b^{\prime}\right) b^{\prime}}{\int_{0}^{\infty} \epsilon\left(b^{\prime \prime}\right) b^{\prime \prime} \mathrm{d} b^{\prime \prime}} \mathrm{d} b^{\prime}=\int_{-\infty}^{d_{625}} P\left(d_{625}^{\prime}\right) \mathrm{d} d_{625}^{\prime}
$$

which gives a one to one monotonous correspondence between $d_{625}$ and $b$ with Jacobian $\mathcal{J}(b)=\left(\frac{\mathrm{d} d_{625}}{\mathrm{~d} b}\right)_{b}$. The final distribution for the single (same with poly) source events, corrected from the efficiency, is obtained as :

$$
P_{\text {single }}^{\text {cor }}(b)=\frac{P_{\text {single }}\left(d_{625}(b)\right) \mathcal{J}(b)}{\epsilon(b)}
$$

The resulting distributions are shown in figure 9 bottom. It can be noticed that, here, we are interested only in the impact parameter distributions and not in the event by event value of the impact parameter. Hence, whereas the events are likely not equally sorted as a function of $d_{625}$ and as a function of $b$ (i.e. the relation $d_{625}(b)$ is not monotonous, and even not functional), the method is still valid. It is enough to suppose that the proportion of single and poly source events along both axes are the same within the Jacobian transform, or, in other words, that the left(right) Gaussian contains only single(poly) source events.

The fusion cross-section is finally evaluated using $\sigma_{\text {single }}=2 \pi \int_{0}^{\infty} P_{\text {single }}^{\text {cor }}(b) b \mathrm{~d} b$, leading to $\sigma_{\text {single }}=170 \pm 20 \mathrm{mb}$ which would correspond to a mean limit impact parameter (sharp cut-off) of $2.3 \mathrm{fm}$ for the considered $\mathrm{Ni}+\mathrm{Ni}$ system at $32 \mathrm{~A} \mathrm{MeV}$, whose total reaction crosssection amounts to $3.8 \mathrm{~b}$ [37]. Note that the detected single-source events correspond to 
about $60 \mathrm{mb}$. Some other measurements of the fusion-like cross-sections, in the same incident energy and nuclei mass ranges, had also given such large values $[31,38]$.

At this stage we wish to remind that the classical trajectory model [39] (used in Simon and DBS) including conservative and dissipative forces (Coulomb potential [40], proximity nuclear interaction [41] and one-body nuclear friction [42]) largely underestimates the fusion crosssection $\left(b_{\text {limit }} \in[1.2,1.4] \mathrm{fm}\right)$. On the other hand, different versions of the BNV [43] model overestimate the cross-section $\left(b_{\text {limit }}>3.5 \mathrm{fm}[7]\right)$. Hence, such experimental measurements are particularly important to validate or constrain the theoretical models. The spread of the measured and predicted sets of fusion cross-section values is due notably to its drastic decrease as a function of the bombarding energy [44].

\section{CONCLUSIONS}

We have shown that, applying for the first time the Discriminant Analysis to the mass/kinetic energy quadri-moments, it was possible to obtain a good separation of singlesource from poly-source events in the system $\mathrm{Ni}+\mathrm{Ni}$ at $32 \mathrm{~A} \mathrm{MeV}$. The resulting discriminating variable $d_{625}$ appears to be robust to the deexcitation mechanism of the single-source nucleus. The events selected using this variable are shown to be representative of the whole set. Such a result could not have been obtained using the traditional global variables. Application to high quality INDRA data has allowed the determination of the fusion probability as a function of the impact parameter. The corresponding cross-section is found to be $\sigma_{\text {single }}=170 \pm 20 \mathrm{mb}$. The impact parameter range where single- and poly-source events coexist goes from $\approx 1$ to $\approx 3 \mathrm{fm}$. The width of this zone is due both to the quantum effects of the partial waves and to the discrimination method's resolution. The result gives an overestimate of the physical coexistence overlap. The characterization of the single-source events selected within the original framework presented here, as well as its evolution with incident energy will be detailed in a forthcoming companion paper [45].

The authors wish to thank X. Artru and D. Wallez for useful discussion. 


\section{REFERENCES}

[1] G.D. Harp, J.M. Miller, B.J. Berae, Phys. Rev. 165 (1968) 1166,

E. Fabrici et al., Phys. Rev. C40 (1989) 2548,

S. Tomasevic, thesis, Université de Strasbourg, IReS T 9546 (1995),

P. Désesquelles et al., Nucl. Phys. A633 (1998) 547.

[2] M. Blann, Phys. Rev. C23 (1981) 205,

M. Blann, Phys. Rev. C31 (1985) 1245.

[3] J.F. Lecolley et al., Phys. Lett. B354 (1995) 202.

[4] N. Marie et al., Phys. Lett. B391 (1997) 15.

[5] M.F. Rivet et al., Phys Lett B430 (1998) 217.

[6] M. D'Agostino et al., Phys. Lett. B368 (1996) 259.

[7] A.M. Maskay, thesis, Université de Lyon, LYCEN T 9969 (1999).

[8] "Treatise on Heavy Ion Science", Vol. 2 Fusion and quasi-fusion Phenomena, Edited by A. Bromley, Plenum Press, New-York (1984).

[9] P. Désesquelles, Ann. Phys. Fr. 20 (1995) p.1 (in English).

[10] M. Mjahed, thèse de $3^{\text {ème }}$ cycle, Université de Clermont 2, PCCF T 8705 (1987).

[11] A.M. Kshirsagar, "Multivariate Analysis", Statistics textbooks and monographs Vol. 2, Marcel Dekker Inc., New York (1972).

[12] D. Durand, Nucl. Phys. A541 (1992) 266.

[13] A.D. NGuyen, thesis, Université de Caen, LPCC T 9802 (1998).

[14] J.P. Bondorf, A.S. Botvina, A.S. Iljinov, I.N. Mishustin, K. Sneppen, Phys. Rep. 257 (1995) 133.

[15] R.J. Charity et al., Nucl. Phys. A483 (1988) 371. 
[16] J. Pouthas et al., Nucl. Instr. and Meth. in Phys. Res. A307 (1991) 325.

[17] E. Lloyd, Handbook of Applicable Mathematics, Volume VI, Statistics, Part B (Wiley, New-York, 1984).

[18] H. Hotelling, Journal of Educational Psychology 24 (1933) 417-441, 498-520.

[19] M. Louvel et al., Nucl. Phys. A559 (1993) 137,

R. Pak et al., Phys. Rev. C53 (1996) R1469,

Y. Larochelle et al., Phys. Rev. C53 (1996) 823,

C. Williams et al., Phys. Rev. C55 (1997) R2132,

J. Barrette et al., Phys. Rev. C51 (1995) 3309,

G.F. Peaslee et al., Phys. Rev. C49 (1994) R2271.

[20] J.D. Frankland, thesis, Université de Paris XI Orsay, IPNO T 9806 (1998).

[21] J. Cugnon, J. Knoll, C. Riedel, Y. Yariv, Phys. Lett. B109 (1982) 167,

J. Cugnon, D. L'Hote, Nucl. Phys. A397 (1983) 519.

[22] D. Cussol, thesis, Université de Caen, GANIL T 8901 (1989), R. Pak et al., Phys. Rev. C54 (1996) 1681.

[23] P. Danielewicz and M. Gyulassy, Phys. Lett. B129 (1983) 283.

[24] E. Galichet, thesis, Université de Lyon I, LYCEN T 9868 (1998).

[25] O. Lopez et al., Phys. Lett. B315 (1993) 34.

[26] R. Bougault et al., Nucl. Phys. A488 (1988) 255c.

[27] J. Lukasik et al., Phys. Rev. C55 (1997) 1906.

[28] P. Désesquelles, Internal report ISN Grenoble, ISN 9640 (1996).

[29] Bao-An Li, A.R. DeAngelis and D.H.E. Gross, Phys. Lett. B303 (1993) 225, P. Kreutz et al., Nucl. Phys. A556 (1993) 672. 
[30] M.T. Magda et al., Phys. Rev. C53 (1996) 1473,

E. Colin et al., Phys. Rev. C57 (1998) 1032.

[31] L. Lebreton et al., Eur. Phys. J. A3 (1998) 325.

[32] C.G. Fox and S. Wolfram, Phys. Rev. Lett. 41 (1978) 1581,

N. Marie, thesis, Université de Caen, GANIL T 9504 (1995).

[33] A. Lleres et al., Phys. Rev. C48 (1993) 2753,

A. Lleres et al., Phys. Rev. C50 (1994) 1973.

[34] P. Désesquelles, J.P. Bondorf, I.N. Mishustin and A.S. Botvina, Nucl. Phys. A604 (1996) 183.

[35] W.H. Press, B.P. Flannery, S.A. Teukolsky, W.T. Vetterling, Numerical Recipes FoRTRAN version, Cambridge University Press (1989).

[36] L. Phair et al., Nucl. Phys. A548 (1992) 489.

[37] S. Kox et al., Nucl. Phys. A420 (1984) 162.

[38] P.F. Box et al., Phys. Rev. C 50 (1994) 934,

A. Fahli et al., Phys. Rev. C 34 (1986) 161.

[39] J.R. Birkelund et al., Phys. Rep. 56 (1979) 107.

[40] J. Bondorf et al., Phys. Rep. C15 (1974) 83.

[41] J. Blocki et al., Ann. Phys. 105 (1977) 427,

J. Blocki et al., Nucl. Phys. A307 (1978) 319.

[42] J. Randrup, Ann. Phys. 112 (1978) 356.

[43] A. Bonasera, F. Gulminelli, J. Molitoris, Phys. Rep. 243 (1994) 1.

M. Colonna, N. Colonna, A. Bonasera, M. Di Toro, Nucl. Phys. A541 (1992) 295.

A. Guarnera, M. Colonna, P. Chomaz, Phys. Lett. B373 (1996) 267. 
[44] P. Lautesse et al., in prep.

[45] A.M. Maskay et al., in prep. 


\section{FIGURES}

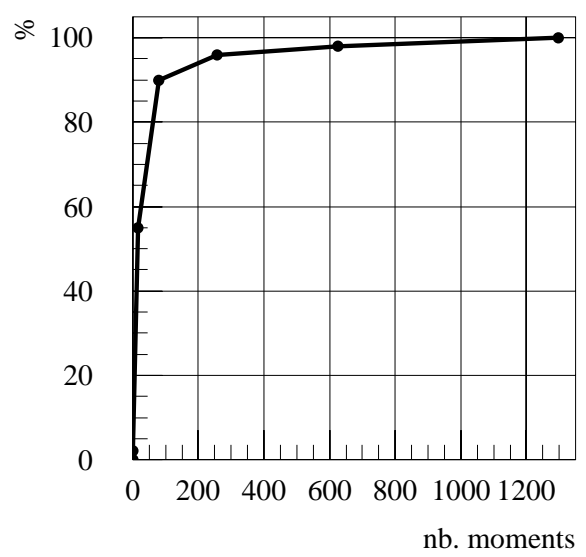

FIG. 1. Percentage of the statistical information given by the first $n^{4}$ quadri-moments relative to the statistical information hold by the first $1296\left(6^{4}\right)$ quadri-moments. 

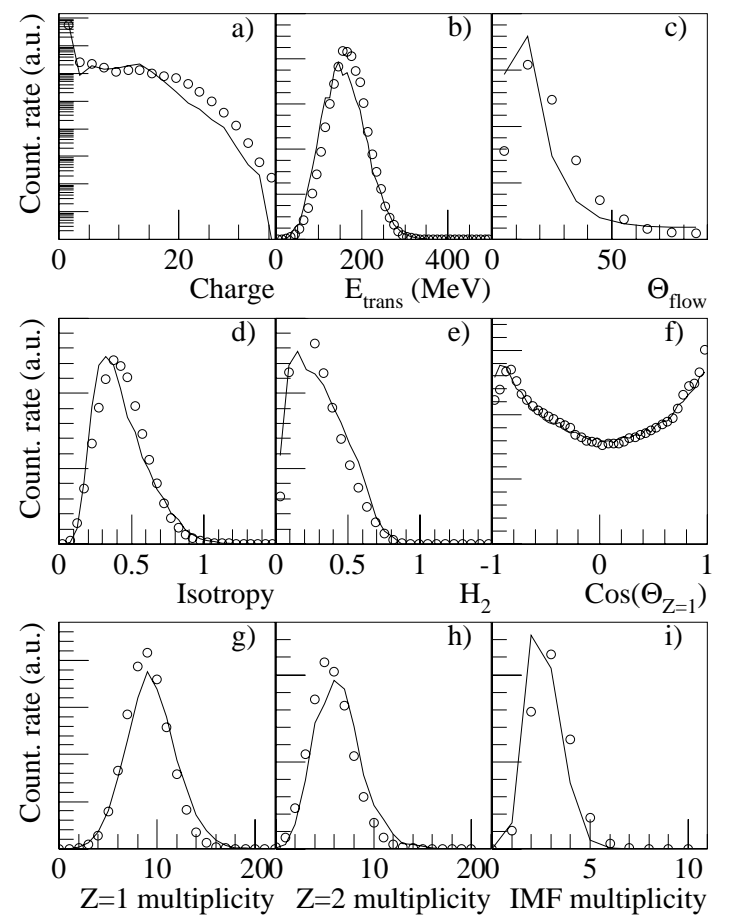

FIG. 2. Comparison of the data generated by the Simon code filtered by the INDRA response function and the completeness condition criteria (lines) with experimental "complete" data (dots) for the $\mathrm{Ni}+\mathrm{Ni}$ at $32 \mathrm{~A} \mathrm{MeV} \mathrm{system.} \mathrm{The} \mathrm{variables} \mathrm{on} \mathrm{the} \mathrm{horizontal} \mathrm{axes} \mathrm{are} \mathrm{:} \mathrm{a)} \mathrm{charge,} \mathrm{b)}$ transverse energy $E_{\text {trans }}=\sum_{\nu} E_{\text {kin } \perp}(\nu), \nu$ running over all the detected products, c) flow angle (calculated with the "energy" tensor), d) isotropy ratio (see Eq. (3)), e) second Fox-Wolfram coefficient, f) cosine of the angle between the hydrogen velocity vector and the beam direction (in the center of mass frame), g) $Z=1$ multiplicity, h) $Z=2$ multiplicity, i) IMF ( $Z \geq 3$ ) multiplicity. See Table I for the definition of the variables. 


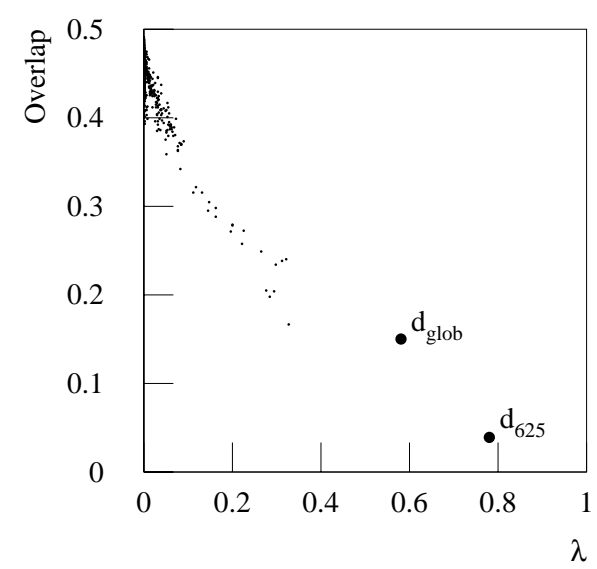

FIG. 3. Overlap $\mathcal{O}$ as a function of the discrimination power $\lambda$ for the 625 quadri-moments (points), their discriminant variable $\left(d_{625}\right.$, see text) and the discriminant variable built on the global variables listed in Table I $\left(d_{g l o b}\right)$. The events are simulated using the Simon code. 


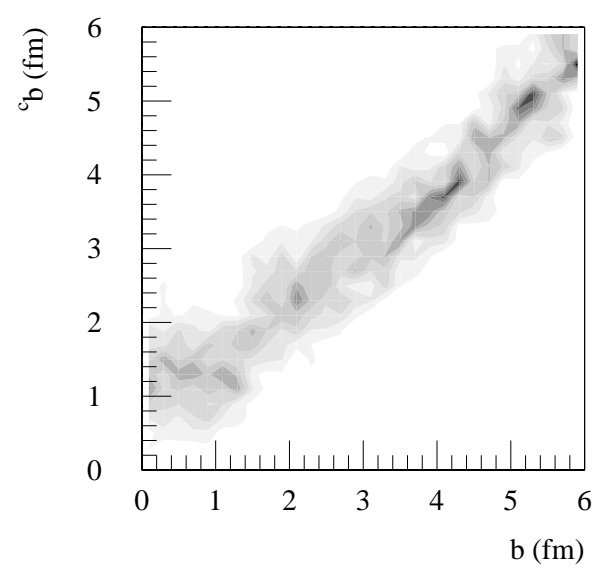

FIG. 4. Correlation $P\left({ }^{c} b \mid b\right)$ between the impact parameter $b$ and its estimate ${ }^{c} b$ obtained as a linear combination of the quadri-moments for the Simon filtered-selected data. 


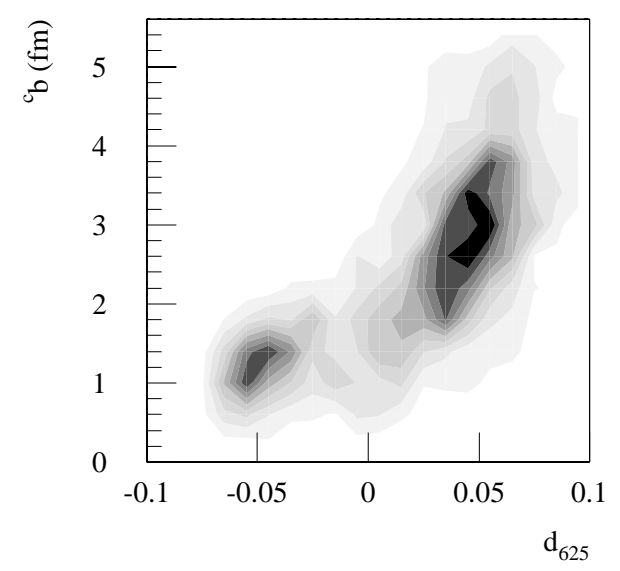

FIG. 5. Correlation between the discriminant variable given by the discriminant analysis on the 625 first quadri-moments and the estimate of the impact parameter for Simon filtered-selected events. The grey scale is linear. 


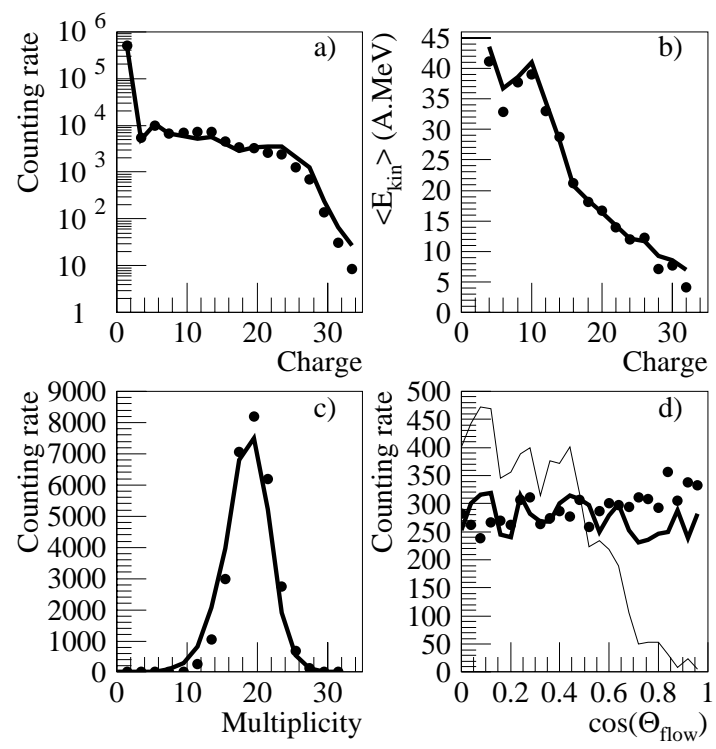

FIG. 6. Representativeness of the selection performed using the discrimination variable $d_{625}$. The dots correspond to the whole set of Simon single-source events and the lines to the sub-set obtained fixing the threshold so that the pollution from poly-source events is $5 \%$ (the lines are normalized to the whole set). a) charge spectrum, b) mean center of mass kinetic energy as a function of the fragment charge, c) charged fragment multiplicity, d) flow angle (the thin line corresponds to the selection obtained using the isotropy ratio). 


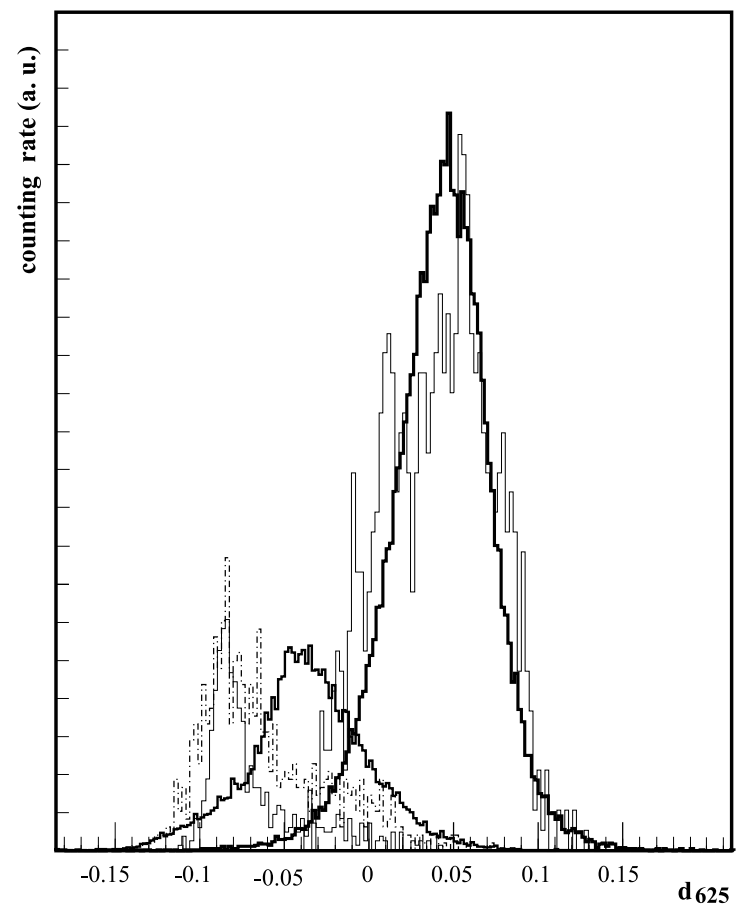

FIG. 7. Distributions of the $d_{625}$ variable obtained with the Simon code. The bold lines stand for the Simon samples (single-source on the left hand side, poly-source on the right-hand side). Comparison is made with DBS+Gemini (thin lines) and DBS+SMM (only in the case of single-source events, dot-dashed line). 


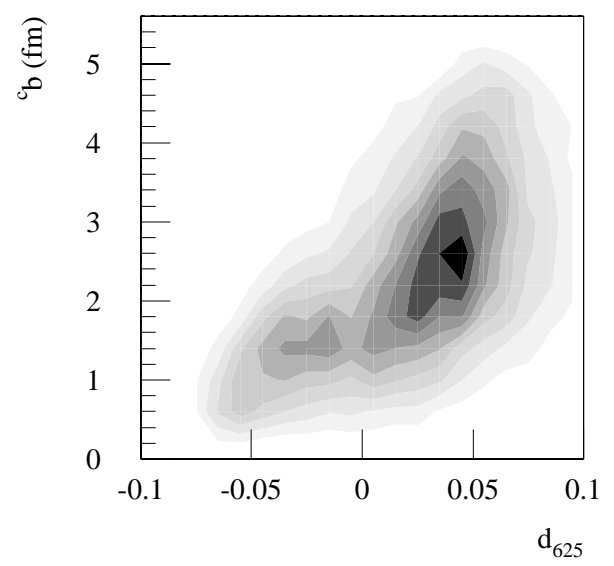

FIG. 8. Same as figure 5 but for experimental data. 

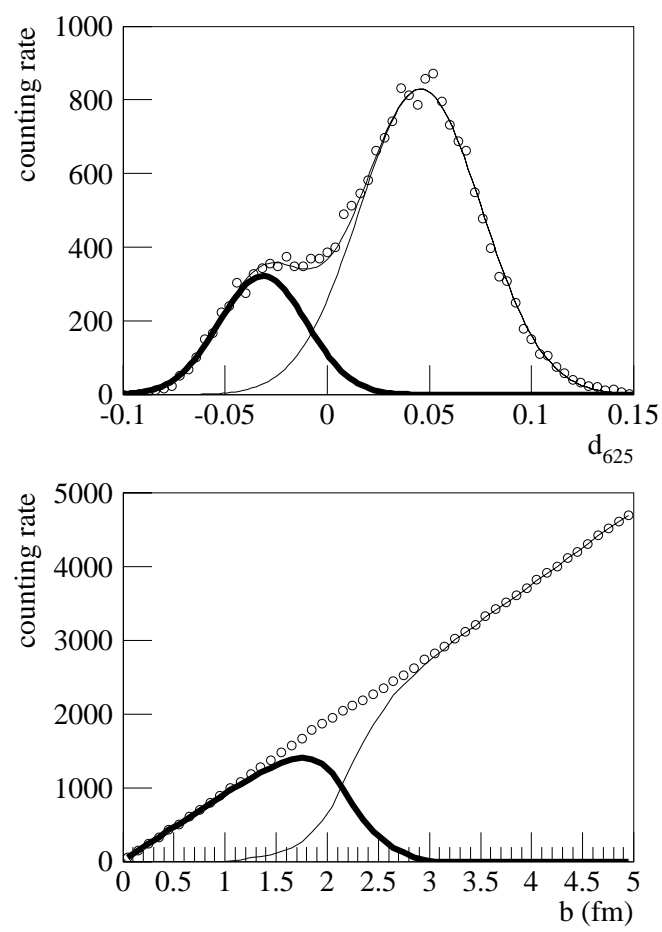

FIG. 9. Top : discriminant variable distribution for the experimental data (dots). The fit by two Gaussians is indicated by a bold line for single-source events and a thin line for poly-source events. Bottom : corresponding impact parameter distributions. 


\section{TABLES}

\begin{tabular}{|c|c|c|}
\hline Global variable name & $\mathcal{O}$ & $\lambda$ \\
\hline Sphericity $^{\dagger}[21]$ & .28 & .21 \\
\hline Sphericity* $\left.{ }^{*} 21\right]$ & .24 & .29 \\
\hline Coplanarity $^{\dagger}[21]$ & .36 & .09 \\
\hline Coplanarity* $[21]$ & .34 & .12 \\
\hline Aplanarity $^{\dagger}[21]$ & .35 & .10 \\
\hline Aplanarity* $[21]$ & .32 & .15 \\
\hline$\Theta_{\text {flow }}^{\dagger}[22]$ & .20 & .48 \\
\hline$\Theta_{\text {flow }}^{*}[22]$ & .20 & .50 \\
\hline${\text { Aspect } \text { ratio }^{\dagger}[23]}^{2}$ & .33 & .04 \\
\hline Aspect ratio* $[23]$ & .30 & .04 \\
\hline Eigenvalue prod..$^{\dagger}[13,24]$ & .31 & .16 \\
\hline Eigenvalue prod.* $[13,24]$ & .27 & .23 \\
\hline$Y_{33}[25,26]$ & .36 & .09 \\
\hline$v_{t o t}^{\|}[25]$ & .44 & .01 \\
\hline$v_{\text {tot }}^{\perp}[25]$ & .46 & .00 \\
\hline$E_{\text {rat }}[13,27]$ & .18 & .46 \\
\hline$\lambda_{\text {event }}[28]$ & .19 & .35 \\
\hline$Z_{12}[29]$ & .28 & .29 \\
\hline Multiplicity $[30,31]$ & .38 & .07 \\
\hline$H_{2}$ (Fox-Wolfram) $[32]$ & .25 & .30 \\
\hline Isotropy ratio $^{1}$ & .19 & .39 \\
\hline Isotropy ratio $^{2}$ & .17 & .54 \\
\hline Disc. var. $d_{g l o b}[9,11]$ & .15 & .58 \\
\hline
\end{tabular}


TABLE I. Values of the overlap and discrimination power in the case of simulated $\mathrm{Ni}+\mathrm{Ni}$ at $32 \mathrm{~A} \mathrm{MeV}$ events for some variables traditionally used in the selection of single-source events. References stand for the definitions of the relevant variables. The variables calculated from the "energy tensor" $\left(T_{i j}=\sum_{\nu} P_{i}(\nu) P_{j}(\nu) /(2 m(\nu))\right)$ are labelled by a dag $\left(^{\dagger}\right)$, the variables calculated from the "momentum tensor" $\left(T_{i j}=\sum_{\nu} P_{i}(\nu) P_{j}(\nu) / P(\nu)\right)$ are labelled by a star $\left(^{*}\right)$. The weighted mean velocity of the detected products is denoted $v_{t o t}$. The event discrimination power $\left(\lambda_{\text {event }}\right)$ measures the separation in velocity space between the quasi-projectile and the quasi-target. The difference between the charges of the two heaviest fragments is denoted $Z_{12}$. The isotropy ratio is calculated in the center of mass with respect to the first ellipsoid eigenvector (label ${ }^{1}$ ) and to the beam axis (label ${ }^{2}$ ). 